\title{
Misturas NR/SBR: Modos de Preparação e Propriedades
}

\author{
Leila L. Y. Visconte, Agnes F. Martins, Regina C. R. Nunes \\ Instituto de Macromoléculas Professora Eloisa Mano, UFRJ
}

\author{
J oão C. M. Suarez \\ Departamento de Engenharia Mecânica e de Materiais, IME
}

Resumo: Aplicações de artefatos de borracha muitas vezes requerem um conjunto de propriedades que não podem ser fornecidas por um único elastômero sendo, então necessário, que misturas de dois ou mais polímeros sejam empregadas. Nesses casos, os demais ingredientes que normalmente são adicionados, distribuem-se de modo diferente dependendo de sua afinidade com cada uma das borrachas. O grau de dispersão de cada um desses ingredientes em cada fase elastomérica irá influenciar a velocidade e o grau de vulcanização e, certamente, terá conseqüências sobre o desempenho do composto final. Neste trabalho, a borracha natural (NR) foi misturada ao elastômero de butadieno-estireno (SBR) na proporção de 1:1 em peso. As composições foram preparadas em misturador de cilindros, segundo a norma ASTM D 3182, empregando-se quatro maneiras distintas para a incorporação dos aditivos. Após a vulcanização foram estudadas propriedades mecânicas, como resistências à tração e ao rasgamento e dureza, propriedades termo-dinâmico-mecânicas e a morfologia de cada uma das composições. As propriedades mecânicas foram avaliadas de acordo com as normas ASTM específicas para cada ensaio. Os resultados mostram que, apesar de se usar a mesma formulação, o modo de preparo tem grande influência sobre as propriedades, o que é detectável pelas propriedades mecânicas e pela análise morfológica, e evidenciado através de testes sensíveis, como o dinâmico-mecânico. No caso da mistura NR/SBR as melhores propriedades são obtidas quando é favorecida a vulcanização da borracha que, isoladamente, apresenta propriedades inferiores.

Palavras-chave: Mistura de elastômeros, propriedades mecânicas, análise termo-dinâmico-mecânica.

\section{NR/SBR Blends: Preparation Modes and Properties}

\begin{abstract}
Frequently a set of properties required for a given application cannot be accomplished by only one elastomer and, thus, mixtures of two or more polymers must be employed. In these cases, the other ingredients normally added are dispersed differently, depending on their affinity to each polymer. The degree of dispersion each additive experiences in each elastomeric phase will influence the rate and the degree of vulcanization and will certainly have consequences on the performance of the final product. In this work, natural rubber (NR) was mixed to butadiene-styrene elastomer (SBR) in a 50:50 w/w ratio. The compositions were prepared in a two-roll mill, according to ASTM D3182, introducing the additives in four different modes. After vulcanization, the mixes were investigated as for their mechanical (stress and tear strengths and hardness), dynamic mechanical thermal and morphological analysis. Mechanical properties were evaluated according to ASTM procedures, specific for each test. The results show that, although having been prepared following the same formulation, the properties of these mixtures are influenced by the preparation mode, which could be detected by mechanical and morphological analysis and evidenced through more sensitive methods as DMTA. For NR/SBR blends, the best properties are achieved when one favours the vulcanization of the rubber with inferior properties, that is, SBR.
\end{abstract}

Keywords: Elastomer blends, mechanical properties, dynamic mechanical thermal analysis.

Autor para correspondência: Leila L. Y. Visconte, Instituto de Macromoléculas Professora Eloisa Mano, UFRJ, C.P. 68525, CEP 21945-970, Rio de Janeiro, RJ.E-mail:Iyv@ima.ufrj.br 


\section{Introdução}

As borrachas natural e sintéticas raramente são utilizadas na forma pura. Para que possam ter aplicação prática, vários são os ingredientes introduzidos, cuja escolha é baseada no conjunto de propriedades desejadas. Muitas vezes, no entanto, o uso de um só elastômero não é suficiente para satisfazer os requisitos de desempenho exigidos para uma dada aplicação.

Um elastômero é misturado a outro por três razões principais: para melhorar as propriedades do elastômero original, melhorar o seu processamento e/ou baixar custos. Todos os elastômeros apresentam deficiência em algum aspecto, de modo que a combinação de dois ou mais elastômeros é uma maneira de se obter um bom balanço de propriedades ${ }^{[1]}$. A mistura resultante é composta de polímeros distintos, com estruturas químicas diferentes e altos pesos moleculares sendo, portanto, total ou parcialmente incompatíveis.

Existem várias maneiras de se realizar a mistura, sendo a mecânica a mais utilizada. A maior dificuldade ao se trabalhar com misturas de borrachas está na obtenção de uma vulcanização uniforme em todas as fases poliméricas, por causa da diferença de solubilidade dos vários aditivos em relação às borrachas utilizadas. Isso significa que os aditivos estarão em maior concentração na fase com a qual têm maior afinidade.

Neste trabalho foram empregadas duas das borrachas mais utilizadas, cujos comportamentos em relação à resistência à tração são bem diferentes. A borracha natural (NR) tem excelente resistência à tração, mesmo na ausência de cargas reforçadoras como o negro de fumo, por causa de sua característica de ser cristalizável sob tração. A borracha de butadienoestireno (SBR) não é cristalizável e apresenta propriedades bem mais modestas sendo, no entanto, a de mais baixo custo e, portanto, a mais utilizada para aplicações gerais em que não se necessita de desempenho específico ${ }^{[2,3]}$. Esses dois elastômeros foram misturados na proporção de 1:1 em peso. Tanto a mistura dos dois elastômeros quanto a incorporação dos demais ingredientes da formulação foram realizadas de quatro maneiras distintas. A partir das composições obtidas foi investigado o efeito do modo de mistura sobre propriedades mecânicas e termo-dinâmico-mecânicas, e sobre a morfologia.

\section{Experimental}

A seguinte formulação foi utilizada na preparação das misturas (em phr): elastômero: NR (50); elastômero: SBR (50); ativador: óxido de zinco $(3,0)$; ativador: ácido esteárico $(2,0)$; antioxidante: octamine, 4,4'-dioctil-difenilamina (2,5); agente de vulcanização: enxofre $(1,5)$; acelerador: TBBS, t-butil2-benzotiazol sulfenamida $(1,1)$. As misturas foram feitas em misturador de cilindros Berstorff, a $50^{\circ} \mathrm{C}$, empregando quatro modos diferentes para a incorporação dos aditivos:

G1: os aditivos, com exceção do acelerador, foram misturados inicialmente na borracha natural e, após homogeneização, foi adicionado SBR. À mistura homogênea, acrescentou-se por último o acelerador.

G2: os aditivos, com exceção do acelerador, foram misturados inicialmente no SBR e, após homogeneização, foi adicionada borracha natural. À mistura homogênea, acrescentou-se por último o acelerador.

G3: as borrachas foram previamente misturadas, antes da adição dos aditivos.

G4: cada uma das borrachas foi misturada com a metade da quantidade de cada um dos aditivos, excetuando-se o acelerador. As composições das duas borrachas foram então misturadas e, após homogeneização, o acelerador foi adicionado.

Após a etapa no misturador de cilindros, procedeu-se à determinação dos parâmetros reométricos utilizando reômetro de disco oscilatório, da marca Tecnología Industrial, modelo TI-100, operando com arco de $3^{\circ}$, numa temperatura de $160^{\circ} \mathrm{C}$.

Os ensaios de tração e rasgamento foram realizados em dinamômetro Instron, segundo as normas ASTM D 412 e D 624, respectivamente, a temperatura ambiente, com velocidade de deformação de 500 $\mathrm{mm} / \mathrm{min}$. Para o ensaio de rasgamento foram usados corpos de prova modelo C. Os corpos de prova foram extraídos de placas, vulcanizadas a partir dos valores de $t_{90}$ (tempo de vulcanização a $90 \%$ de cura).

A análise termo-dinâmico-mecânica foi realizada no analisador DMTA, da marca Rheometric Scientific, modelo MK III, frequiência de $1 \mathrm{~Hz}$, velocidade de aquecimento de $2^{\circ} \mathrm{C} / \mathrm{min}$, modo flexão e faixa de temperatura de -80 a $10^{\circ} \mathrm{C}$. 
A morfologia das misturas foi analisada por microscopia eletrônica de varredura (MEV), utilizando-se um microscópio Jeol, modelo JSM 5800 LV. A análise morfológica foi realizada em superfícies de fratura resultantes da quebra mecânica das amostras, após sua imersão em nitrogênio líquido. $\mathrm{O}$ exame microscópico foi feito após o recobrimento das superfícies de fratura das amostras com fina camada de ouro, em câmara a vácuo.

\section{Resultados e Discussão}

Resultados de propriedades mecânicas podem ser vistos nas Figuras 1 e 2, para cada uma das borrachas isoladamente e para as quatro misturas.

A Figura 1 mostra que os valores de dureza para as misturas são intermediários aos das borrachas puras e não variam significativamente com o modo de preparo das misturas, como já era esperado.

Comparando-se as resistências à tração e ao rasgamento, apresentadas na Figura 2, observa-se que o comportamento das misturas, com relação a essas duas propriedades, não é igual. As misturas G1 e G3 apresentaram valores de resistência ao rasgamento semelhantes ao da NR, enquanto G4 foi a de pior resultado. O melhor valor foi obtido para G2, superior até ao valor obtido para a NR.

Quanto à resistência à tração, comprova-se que o valor desta propriedade para a NR é muito superior ao do SBR e que, para as misturas, os valores são intermediários aos das duas borrachas isoladas. Estes valores, no entanto, dependem do modo de preparo. A

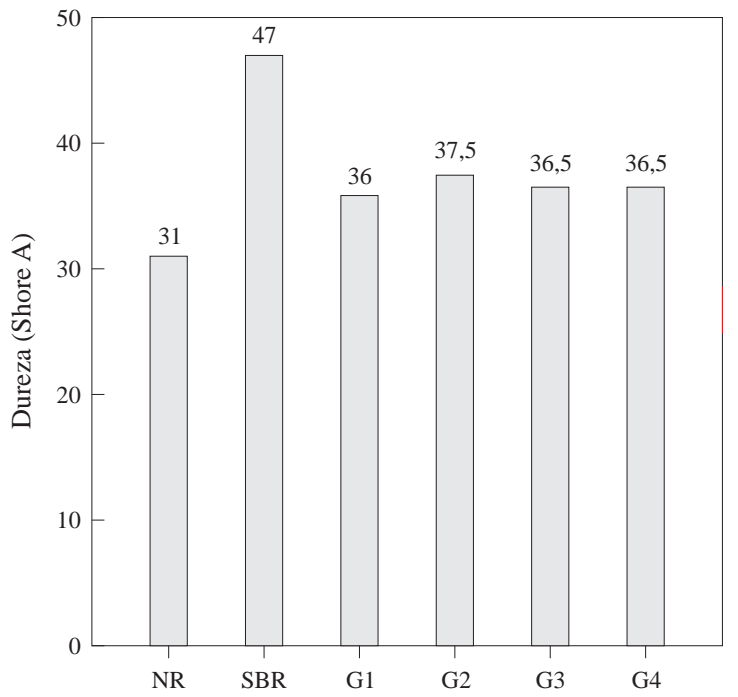

Figura 1. Dureza Shore de misturas NR/SBR

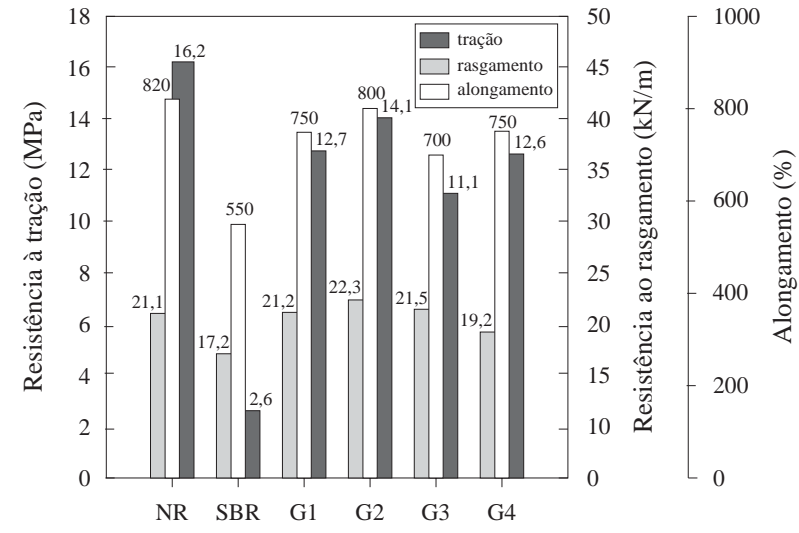

Figura 2. Propriedades mecânicas de misturas NR/SBR

resistência à tração para $\mathrm{G} 2$ é maior que para $\mathrm{G} 1$ e pior para G3. Os resultados para G1 e G4 são praticamente iguais, mostrando que aumentando-se a concentração do sistema de vulcanização, o processo de vulcanização não é, necessariamente, favorecido no sentido de melhorar a resistência à tração. No caso dos elastômeros NR e SBR, cujas propriedades diferem grandemente, o desafio é favorecer aquela de pior desempenho, ao mesmo tempo em que se procura preservar a outra.

A Tabela 1 mostra os parâmetros reométricos para as borrachas puras e para as quatro misturas.

Comparando-se as composições contendo as borrachas isoladas observa-se que, devido à estrutura química da NR, há um favorecimento maior para a formação de ligações cruzadas nesta borracha, o que corrobora as diferenças encontradas quando se compara NR com SBR. Já para as composições com mistura de borrachas, tem-se que os parâmetros reométricos para as quatro composições estudadas são semelhantes, com pequena diferença para a composição G4.

As curvas reométricas para as diferentes composições apresentadas na Figura 3 mostram que o

Tabela 1. Propriedades reométricas de misturas NR/SBR

\begin{tabular}{ccccc}
\hline Material & $\begin{array}{c}\mathbf{t}_{\mathbf{9 0}} \\
(\mathbf{m i n})\end{array}$ & $\begin{array}{c}\mathbf{M}_{\mathbf{L}} \\
(\mathbf{l b . i n})\end{array}$ & $\begin{array}{c}\mathbf{M}_{\mathbf{H}} \\
(\mathbf{l b . i n})\end{array}$ & $\begin{array}{c}\mathbf{t}_{\mathbf{s 1}} \\
(\mathbf{m i n})\end{array}$ \\
\hline NR-goma & 5,25 & 8,0 & 41,3 & 1,25 \\
SBR-goma & 21,5 & 6,0 & 30,8 & 2,0 \\
G1 & 17 & 5,5 & 46 & 2,25 \\
G2 & 17,5 & 5,0 & 46,5 & 1,25 \\
G3 & 17 & 5,5 & 46 & 1,0 \\
G4 & 18,5 & 4,5 & 46 & 1,0 \\
\hline
\end{tabular}

$\mathrm{t}_{90}:$ tempo ótimo de cura; $\mathrm{M}_{\mathrm{L}}$ : torque mínimo;

$\mathrm{M}_{\mathrm{H}}$ : torque máximo; $\mathrm{t}_{\mathrm{s} 1}$ : tempo de pré-vulcanização 
sistema de vulcanização usado neste trabalho ${ }^{[4]}$, isto é, óxido de zinco e ácido esteárico como ativadores e TBBS como acelerador, desfavorece composições com NR, já que para esta composição o torque atinge um valor máximo, correspondente ao máximo de vulcanização, o qual em seguida decresce, como resultado do início de degradação.

A degradação termo-oxidativa para as borrachas ocorre por dois mecanismos ${ }^{[2]}$. O primeiro deles passa pela cisão ou despolimerização do elastômero, que torna o elastômero gradativamente mais macio e pegajoso. No segundo mecanismo, a oxidação das ligações duplas carbono-carbono dá origem a radicais livres que, por sua vez, podem atacar as cadeias do polímero, levando a um aumento do número de ligações cruzadas. Neste caso, o resultado é a transformação do material elastomérico em um material duro e quebradiço.

Neste trabalho, as misturas NR/SBR apresentam valores de torque máximo, relacionado ao número de ligações cruzadas, superiores aos das borrachas isoladas. Observa-se também, a partir da Figura 3, que a velocidade de vulcanização para a NR é maior que para as misturas e bem maior que para o SBR. Isso significa que os tempos ótimos de cura $\left(\mathrm{t}_{90}\right)$ usados na vulcanização das misturas são excessivamente altos, podendo então provocar degradação parcial da fase NR. Entretanto, como não se observa queda no torque máximo, pode-se supor que ou a fase NR está sofrendo degradação via o segundo mecanismo, ou estaria havendo, de certa forma, uma ação protetora do SBR que, na mistura, dificultaria a degradação da NR, ou ambos os casos. Como, de acordo com a literatura ${ }^{[5]}$, a degradação da NR acontece normalmente segundo o primeiro mecanismo, presume-se que nos casos em que a vulcanização da NR é fortemente favorecida, os produtos da cisão podem estar auxiliando na vulcanização do

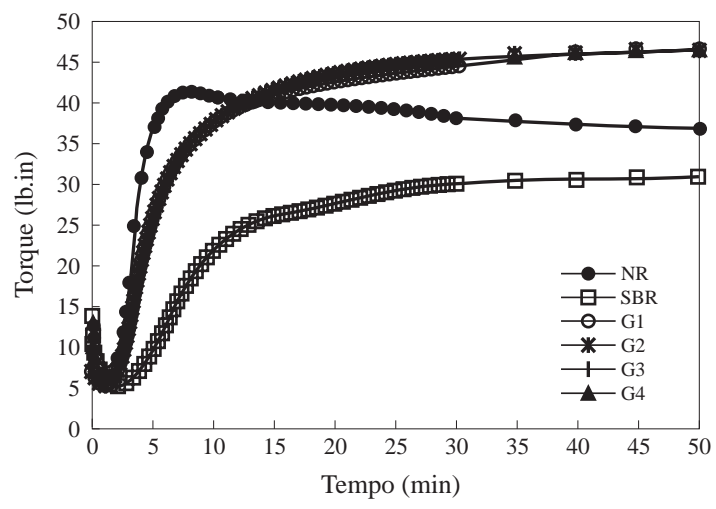

Figura 3. Curvas reométricas de misturas NR/SBR
SBR. Entretanto, a vulcanização excessiva da NR pode levar a um alto grau de degradação desta borracha, comprometendo o desempenho mecânico da mistura.

Dessa maneira, quanto menos se favorecer a vulcanização da fase NR nas misturas, melhores deverão ser os resultados mecânicos.

Um outro ponto a ser considerado é a maior afinidade dos aditivos em relação à NR, o que faz com que esses compostos se encontrem dispersos preferencialmente nesta fase. A afinidade dos aditivos em relação aos diferentes elastômeros, é um tema bastante discutido na literatura ${ }^{[6-12]}$. Assim, a mistura G3, na qual os aditivos foram adicionados à mistura de borrachas, as propriedades mecânicas ficaram prejudicadas tanto pela possibilidade de haver uma maior concentração dos vulcanizantes na fase NR, quanto pelo alto valor de $t_{90}$, favorecendo um alto grau de vulcanização desta borracha e a sua conseqüente degradação.

A incorporação da NR ao elastômero SBR foi vantajosa do ponto de vista das propriedades mecânicas pois a resistência ao rasgamento não foi prejudicada, embora tenha havido uma pequena perda na resistência à tração, mas principalmente pelas propriedades reométricas, como pode ser visto. Além disso há o aspecto econômico uma vez que as composições obtidas apresentarão preços mais competitivos, em face do custo mais baixo do SBR em relação à NR.

Os resultados da análise termo-dinâmico-mecânica podem ser vistos na Figura 4, na qual aparecem as regiões em que ocorrem as transições correspondentes à fase NR (a temperaturas mais baixas) e aquelas correspondentes à fase SBR.

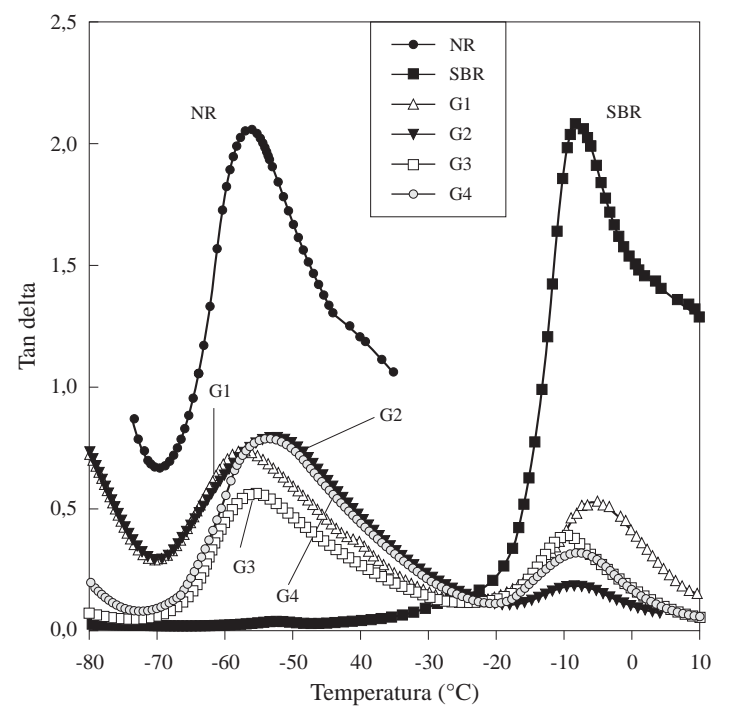

Figura 4. Tan $\delta$ versus temperatura de misturas NR/SBR 
Segundo a literatura ${ }^{[13]}$, graus crescentes de vulcanização levam a uma diminuição nos valores de $\tan \delta$.

Os valores de resistência à tração mostraram que a mistura G3 apresentou o pior resultado. Como os aditivos foram incorporados às borrachas já misturadas, eles puderam migrar preferencialmente para a fase com a qual tinham mais afinidade, isto é, NR. Desse modo, grande parte dos agentes de cura foi consumida na vulcanização da NR, o que explica o baixo valor de $\tan \delta$ para esta fase, e alto valor para a fase SBR. Além disso, o baixo valor de $\mathrm{T}_{\mathrm{g}}$ para a fase NR está relacionado à sua degradação, devido ao alto tempo de vulcanização necessário à mistura. $\mathrm{O}$ mesmo comportamento da $\mathrm{T}_{\mathrm{g}}$ é verificado para $\mathrm{G} 1$, em que todos os ingredientes foram incorporados à fase NR, colaborando para uma cura excessiva desta fase. Em relação a G2 e G4, como a vulcanização da fase SBR foi favorecida, foram obtidos resultados melhores de propriedades mecânicas, uma vez que a fase NR foi razoavelmente preservada, isto é, não foi excessivamente vulcanizada. Isso é sugerido pelos valores de $\tan \delta$, os mais altos e próximos entre si,

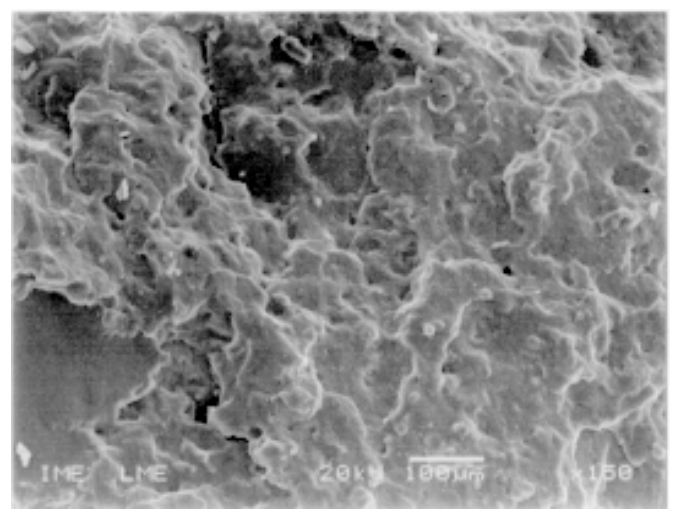

(a)

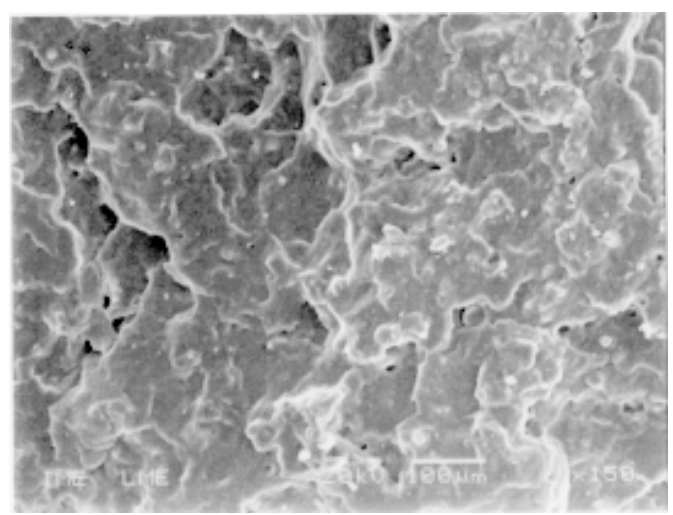

(c) bem como pelos maiores valores de $\mathrm{T}_{\mathrm{g}}$ para a fase $\mathrm{NR}$

Considerando a região correspondente ao $\mathrm{SBR}$, o menor grau de vulcanização desta fase é apresentado por G1, enquanto que para G2 esta fase foi a mais vulcanizada.

A Figura 5 (a)-(d) mostra as fotomicrografias das amostras criofraturadas, e pode ser observado que todas as misturas apresentaram aspecto semelhante - uma morfologia compacta e homogênea. O exame com maiores aumentos (Figura 6 (a)-(d)) permite detectar a existência de pequenas partículas distribuídas na matriz, e que são mais acentuadas em G3 e G4 resultantes, provavelmente, de uma homogeneização deficiente dos aditivos. Estas partículas poderão funcionar como concentradores de tensão, reduzindo a resistência mecânica das misturas (Figura 2). Verifica-se, ainda, a existência de zonas de rasgamento em G2 e G3, indicando que estas misturas apresentam uma maior plasticidade. Pela Figura 2 pode ser comprovada a maior plasticidade de G2, bem como diminuição desta propriedade para G3 devido à má distribuição dos aditivos, explicada anteriormente.

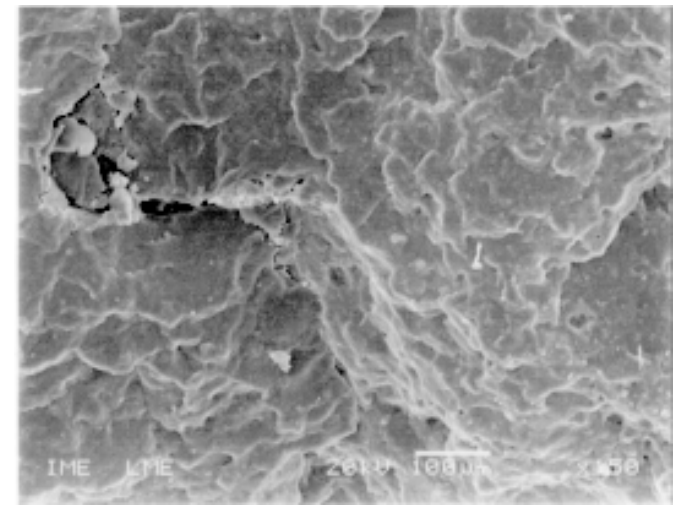

(b)

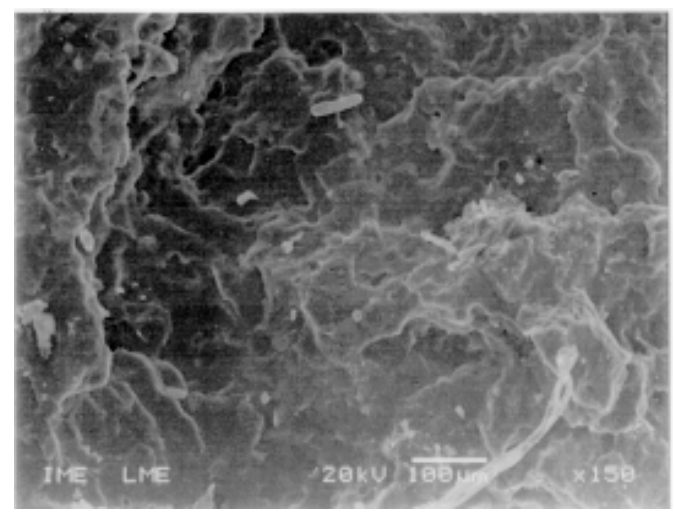

(d)

Figura 5. Fotomicrografias de misturas NR/SBR, 20 kV, 150X: (a) G1; (b) G2; (c) G3; (d) G4 


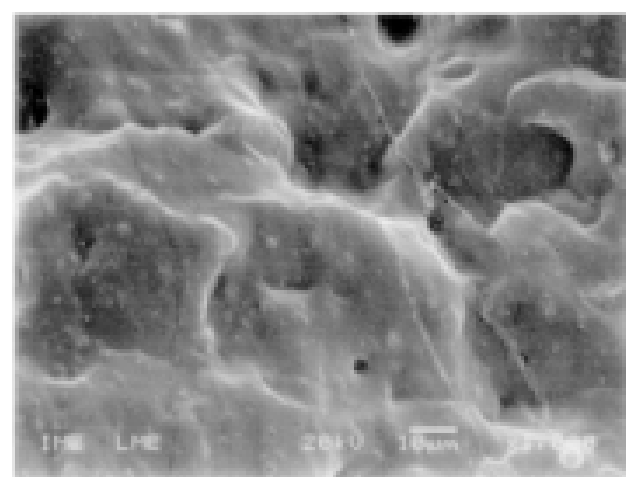

(a)

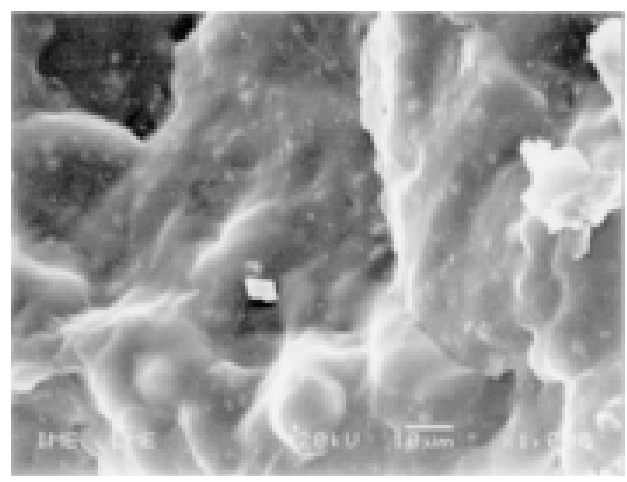

(c)

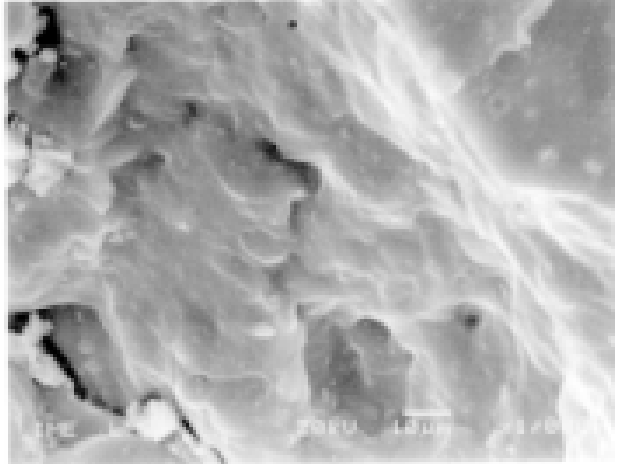

(b)

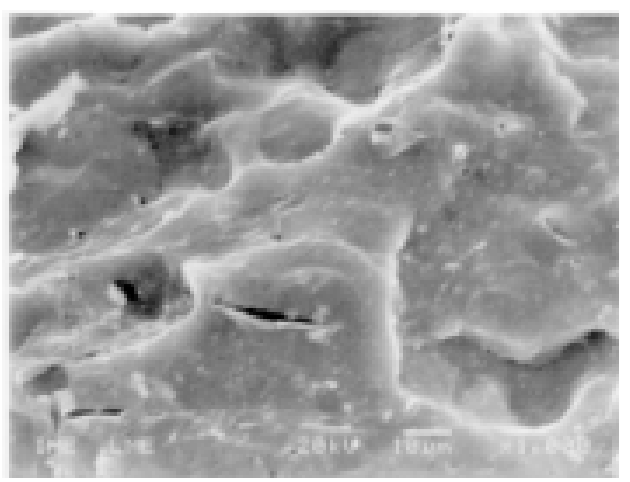

(d)

Figura 6. Fotomicrografias de misturas NR/SBR, 20 kV, 1000X; (a) G1; (b) G2; (c) G3; (d) G4

\section{Conclusão}

A maneira como os aditivos são incorporados em uma mistura de borrachas influencia suas propriedades mecânicas. Se os componentes elastoméricos têm características muito diferentes, como é o caso da mistura NR/SBR, as melhores propriedades são obtidas favorecendo-se a vulcanização da borracha que, quando isolada, apresenta propriedades inferiores, ao mesmo tempo em que se procura preservar as boas qualidades da outra.

\section{Referências Bibliográficas}

1. Corish, P. J. - "Elastomer Blends", in: Science and Technology of Rubber, J. E. Mark, B. Herman e F. R. Eirich (ed.), Academic Press, Inc., San Diego, Califórnia (1994).

2. Brydson, J.A. - "Natural Rubber" in: Rubbery Materials and Their Compounds, Elsevier Applied Science (ed.), London (1988).

3. Brydson, J. A. - "Styrene-Butadiene Rubbers" in: Rubbery Materials and Their Compounds, Elsevier Applied Science (ed.), London (1988).
4. Wampler, W. A.; Gerspacher, M.; Yang, H.H. \& O’Farrell, C.P. - paper $n^{\circ} 52$ presented to Rubber Division, American Chemical Society, Pittsburgh, Pennsylvania, October 1994.

5. Morton, M. - "Introduction to Polymer Science", in: Rubber Technology, M. Morton (ed.), Van Nostrand Reinhold, New York (1987).

6. Kraus, G. - J. Appl. Polym. Sci., 7, p.861 (1963).

7. Porter, M. - Rubber Chem. Technol., 40, p.866 (1967).

8. Mukhopadhyay, R.; De, S. K. - Rubber Chem. Technol., 52, p.263 (1979).

9. Boonstra, B. B.; Taylor, G.L. - Rubber Chem. Technol., 38, p.943 (1965).

10. Bhowmick, A. K.; De, S. K. - Rubber Chem. Technol., 52, p.985 (1979).

11. Bhowmick, A. K.; De, S. K. - Rubber Chem. Technol., 53, p.960 (1980).

12. Pranab K. P.; De, S. K. - J. Appl. Polym. Sci., 28, p.3333 (1983).

13. Huson, M. G. \& McGill, W. J. - J. Polym. Sci.: Polym. Lett. Ed., 22, p.143 (1984).

Recebido: $26 / 03 / 01$

Aprovado: 01/08/01

Polímeros: Ciência e Tecnologia, vol. 11, no 2, p. 76-81, 2001 